\title{
Presencia espacial y temporal de Aramus guarauna (Gruiformes: Aramidae) y de Rostrhamus sociabilis (Accipitriformes: Accipitridae) en la Reserva de la Biósfera Marismas Nacionales, Nayarit, México
}

\author{
Roberto Carmona ${ }^{1}$, Luis Francisco Mendoza ${ }^{1}$, David Molina ${ }^{1}$, Paulina Martínez ${ }^{2}$, Jonathan Vargas ${ }^{2}$ y Miguel Cruz ${ }^{3}$ \\ 1. Laboratorio de Aves. Posgrado en Ciencias Marinas y Costeras. Departamento de Biología Marina, Universidad Autónoma de Baja California Sur, \\ A.P. 19-B, La Paz, Baja California Sur, 23080, México; beauty@uabcs.mx \\ 2. Unidad Académica de Agricultura. Universidad Autónoma de Nayarit. Km. 9 carretera Tepic-Compostela. Xalisco, Nayarit; \\ pmartinezsarabia@gmail.com \\ 3. Programa de Conservación de Aves. Pronatura Noroeste, Ensenada, Baja California, México; mcruz@pronatura-noroeste.org
}

Recibido 30-VIII-2012 Corregido 28-X-2012 Aceptado 23-XI-2012

\begin{abstract}
Spatial and temporal presence of Aramus guarauna (Gruiformes: Aramidae) and Rostrhamus sociabilis (Accipitriformes: Accipitridae) in the Marismas Nacionales Biosphere Reserve, Nayarit, Mexico. Monthly visits were conducted at 12 sites located at Marismas Nacionales (November 2010 to June 2011). In five of them we regularly observed the Limpkin (Aramus guarauna) and in three, the Snail Kite (Rostrhamus sociabilis). The Apple Snail (Pomacea spp.) is abundant in the brackish wetlands and is the most important prey of both species. In 31 observations we recorded 493 limpkins, mainly in Las Cañadas and La Batanga (together: $64 \%$ of the records). In March and April we observed flocks of up 80 individuals, in probable relation with the dry season. We obtained 109 records of the kites, always lonely and concentrated in Zoquipan and Mexcaltitan (96\%). The presence of the snail in Marismas Nacionales allows both species to expand their range along the Mexican Pacific, at least to the north of Sinaloa, were the lack of fresh water will hinder the establishment of snail populations.
\end{abstract}

\section{KEY WORDS}

Aramus guarauna, Rostrhamus sociabilis, spatial distribution, temporal distribution, Marismas Nacionales, Nayarit, Pomacea spp.

\section{RESUMEN}

Realizamos visitas mensuales a 12 sitios ubicados en la Reserva de la Biósfera Marismas Nacionales (noviembre 2010 a junio 2011), en cinco de ellos observamos regularmente carraos (Aramus guarauna) y gavilanes caracoleros (Rostrhamus sociabilis) en tres de estos. En las zonas de observación (humedales salobres) abundó el caracol manzana (Pomacea spp.), la presa más importante de ambas especies. Para el carrao se obtuvo 493 registros en 31 eventos de observación, los sitios de mayor importancia fueron Las Cañadas y La Batanga (en conjunto 64\% de los registros); en marzo y abril observamos parvadas de hasta 80 individuos, en probable relación con la época de estiaje. Del gavilán obtuvimos 109 registros, de individuos solitarios; los sitios más importantes para esta especie fueron Zoquipan y Mexcaltitán (96\%). Dada la presencia de los caracoles manzana, Marismas Nacionales es utilizada regularmente por ambas especies y éstas pueden continuar expandiendo su intervalo de distribución a lo largo del Pacífico mexicano, al menos hasta el norte de Sinaloa, donde la falta de agua dulce y vegetación acuática dificultará el establecimiento de poblaciones de caracoles.

\section{PALABRAS CLAVE}

Aramus guarauna, Rostrhamus sociabilis, distribución espacial, distribución temporal, Marismas Nacionales, Nayarit, Pomacea spp.
El Carrao (Aramus guarauna Linnaeus, 1766) y el Gavilán Caracolero (Rostrhamus sociabilis Vieillot, 1817) son dos especies tropicales que basan su alimentación principalmente en caracoles dulceacuícolas (Pomacea spp. Perry, 1810), por lo que la presencia de ambas especies está estrechamente relacionada con la de su principal recurso alimenticio (Sykes, Rodgers \& Bennetts, 1995; Bryan, 2002;
Iliff \& Lovitch, 2007). Ambas especies se encuentran protegidas por el Gobierno Mexicano, el Carrao como amenazado y el Gavilán Caracolero bajo protección especial (DOF, 2010).

En México su distribución original, hasta inicios de los años noventa del siglo pasado, era muy similar e incluía desde la península de Yucatán hasta el centro de Veracruz, 
con una pequeña conexión al sur de las costas de Oaxaca hasta El Salvador para el Carrao, y otra a través del Istmo de Tehuantepec a la vertiente del Pacífico (Oaxaca) para el Gavilán Caracolero (Howell \& Webb, 1995).

Sin embargo, recientemente se ha notado la presencia de estas especies en diferentes estados del oeste del país, lo cual se ha relacionado a la previa colonización de los cuerpos de agua por los caracoles manzana (Pomacea spp.). El origen de la llegada de los caracoles es incierto, aparentemente se puede relacionar a programas acuaculturales recientes, como sucedió en Jalisco (PalomeraGarcía, Contreras-Martínez, Cruz-Rivera, Villa-Bonilla \& Gómez-Llamas, 2006). De ser así se trataría de la especie $P$. flagellata Say, 1827, aunque existe un dato en la literatura que indica que $P$. patula Baker, 1992 fue introducido hace cerca de 40 años desde Veracruz en los humedales dulceacuícolas de Jalisco, Colima y Michoacán (JaimeVargas, 1992 en García-Ulloa, Rammarine, Ponce-Palafox \& Góngora-Gómez, 2006).

Los primeros registros del Carrao para la costa centro occidental de México se llevaron a cabo en Manzanillo, Colima en 1994 (Howell, 1994). Para Jalisco el primer registro se publicó en 1999 (Hernández-Vázquez, Esparza, Durand \& Alvarado, 1999). Posteriormente el número y lugares de registro se han visto incrementados, tanto para Jalisco (Palomera-García et al., 2006) como para Colima y Nayarit (Gómez de Silva, 2002; Howell, 2004; Iliff \& Lovitch, 2007; Molina, 2008). En San Blas, al sur de Nayarit, la especie fue reportada en el 2000 (Gómez de Silva, 2002; Howell, 2004), donde actualmente es común observarla (Iliff \& Lovitch, 2007).

El Gavilán Caracolero aparentemente llegó a estas zonas posterior al Carrao, pues no fue reportado en los primeros trabajos (Howell, 1994), pero ha sido enlistado en los siguientes (Howell, 2004; Palomera-García et al., 2006; Iliff \& Lovitch, 2007), de hecho ya han sido registrados nidos de esta especie en Manzanillo, Colima (Palomera-García et al., 2006). De tal forma se ha indicado la posibilidad de que estas especies continúen expandiendo su intervalo de distribución en el oeste de México (Iliff \& Lovitch, 2007).

En este trabajo se presentan los resultados sobre la distribución espacial y temporal del Carrao y del Gavilán Caracolero obtenidos de ocho visitas sistemáticas mensuales realizadas en 12 localidades ubicadas dentro de la Reserva de la Biósfera de Marismas Nacionales (norte de Nayarit y sur de Sinaloa).

\section{METODOLOGIA}

\section{Área de estudio}

Marismas Nacionales es un extenso sistema de humedales que se localiza al norte de Nayarit y sur de Sinaloa, en la costa noroccidental del Pacífico mexicano $\left(21^{\circ} 30^{\prime} 46^{\prime \prime}\right.$ $22^{\circ} 49^{\prime} 18^{\prime \prime} \mathrm{N}$, y $105^{\circ} 38^{\prime} 40^{\prime \prime}-105^{\circ} 27^{\prime} 52^{\prime \prime} \mathrm{W}$; Fig. 1). Tiene una superficie de 300 000ha, de las cuales 133 854ha están incluidas dentro de la Reserva de la Biósfera. El uso de suelo en la región es predominantemente agropecuario. La zona es una red de lagunas costeras salobres, manglares, planicies lodosas, cañadas y marismas (Ortega-Solís, 2011). La Comisión Nacional para el Conocimiento y Uso de la Biodiversidad considera a Marismas Nacionales como una Región prioritaria bajo tres rubros: Marina, Terrestre e Hidrológica (CONANP, 2005).

La zona presenta un clima cálido subhúmedo con lluvias en verano. Las lluvias son abundantes y rara vez inferiores a los $1000 \mathrm{~mm}$ anuales, inician en mayo, aunque

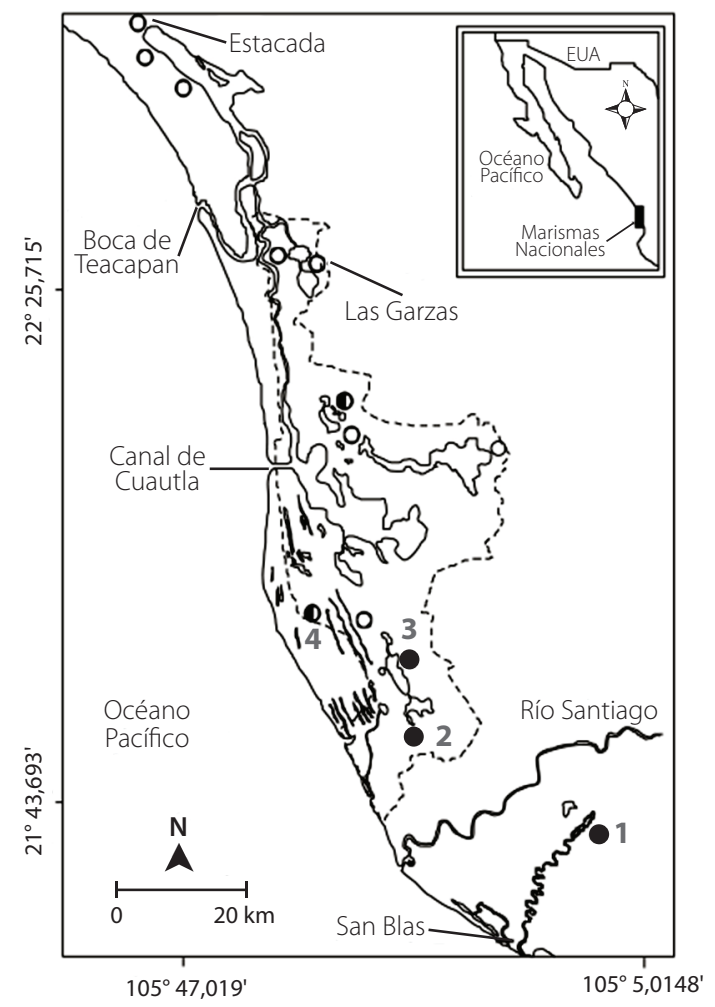

FIG. 1. Área de estudio. Se indican los sitios de observación (círculos llenos = sitios visitados donde se registraron Carraos y Gavilanes Caracoleros; círculos medios = sitios donde sólo se observaron Carraos y círculos vacíos = sitios visitados donde no se observaron estas especies). (1) Zoquipan, (2) La Batanga, (3) Mexcaltitán, (4) Las Cañadas y (5) Zoyata. 
se concentran de junio a octubre, época en las que en promedio precipitan $964 \mathrm{~mm}$ (92\%), en los siete meses restantes llueve un promedio de $81 \mathrm{~mm}$ (CONAGUA, 2004; INEGI, 2011). La vegetación predominante es de bosque de manglar, vegetación halófita rastrera (Salicornia spp. Linnaeus, 1753 y Batis maritima Linnaeus, 1759), selva baja perennifolia, palma de aceite (Orbignya spp. Martius, 1837), selva baja caducifolia, pastizales, potreros, cultivos de frutales y hortalizas y vegetación secundaria, entre otras. La vegetación acuática está representada por lirio acuático (Eichhornia crassipes Solms, 1883), sauces (Salix spp. Linnaeus, 1753) y tule (Typha latifolia Linnaeus, 1753).

El sistema es tan complejo que resulta imposible recorrerlo en su totalidad. Por lo anterior, con base a dos visitas prospectivas realizadas en septiembre y octubre de 2010 y a un reconocimiento aéreo llevado a cabo en enero de 2011 se seleccionaron 12 áreas importantes para las aves acuáticas, incluidos los Carraos y los Gavilanes Caracoleros (Fig. 1). En cinco de ellas se registró la presencia de Carraos (Zoquipan, La Batanga, Mexcaltitán, Las Cañadas y Zoyata; Fig. 1) y en tres de éstas se observaron Gavilanes Caracoleros (Zoquipan, La Batanga y Mexcaltitán; Fig. 1).

Zoquipan es un sistema lagunar adyacente a San Blas (Fig. 1); incluye un canal principal y cuatro cuerpos de agua, dos de ellos estacionales. El canal principal desemboca en el Océano Pacífico. Presenta un gradiente de salinidades que va de la boca (altas salinidades) hacia el interior del sistema. Su vegetación incluye manglares (Rhizophora mangle Linnaeus, 1753), que cubren el perímetro de los canales y la mayor parte de las lagunas, donde también existe zonas bordeadas por tule (Typha latifolia). El lirio (Eichhornia crassipes) cubre una extensa superficie del agua. El resto de la vegetación es selva baja caducifolia, selva mediana caducifolia, vegetación halófita y popal (INEGI, 2000).

La Batanga es una laguna salobre estacional (Fig. 1) que se comunica con otros cuerpos de agua por medio de canales de poca profundidad, por donde puede tener aporte de agua salada. La vegetación predominante es el manglar que presenta importantes extensiones y cubre los canales, la vegetación halófita (Salicornia spp. y Batis maritima) bordea el perímetro de la laguna y parte del área es utilizada como potrero.

La zona de Mexcaltitán (Fig. 1) comprende una extensa laguna continental (902ha), que es el cuerpo principal y otras más pequeñas localizadas en sus adyacencias. La profundidad es baja (menor a $1 \mathrm{~m}$ ) en la mayor parte de su extensión. La vegetación predominante es el manglar; el lirio cubre amplias áreas del espejo de agua. La salinidad es muy baja en la época de lluvias y se incrementa en secas y durante mareas vivas.
Las Cañadas es uno de los complejos estuarinos más grande en la costa del Pacífico mexicano (Kovacs, Zhang \& Flores-Verdugo, 2008; Fig. 1). El sistema cuenta con numerosas lagunas salobres de poca profundidad, formadas por la presencia de aproximadamente 150 barras de arena semiparalelas, en las que existe vegetación de manglar (Avicennia germinans Linnaeus, 1764 y Laguncularia racemosa L. Gaetner, 1805) y halófita (Salicornia spp. y Batis maritima). El impacto humano es notorio en la mayoría de las barras, pues son utilizadas como áreas de cultivos y como zona de pastoreo.

La zona de Zoyata (Fig. 1) está formada por tres lagunas continuas de escasa profundidad (menor a $1 \mathrm{~m}$ ) en la mayor parte de su extensión, pero llegan a los $2 m$ en los canales, muchos de los cuales son dragados para facilitar el tránsito de embarcaciones. La vegetación está conformada principalmente por manglares y tulares. Durante la temporada de lluvias estas lagunas presentan salinidades bajas pero conforme se drenan la salinidad aumenta.

\section{Método}

Las zonas elegidas se visitaron mensualmente entre noviembre de 2010 y junio de 2011. En cada visita se formaron dos equipos de trabajo, uno de los cuales recorrió el sistema desde el norte de Marismas Nacionales (La Estacada) y el otro desde el sur (Laguna de Zoquipan), para encontrarse en Laguna Las Garzas (Fig. 1). Cada equipo de trabajo se conformó con al menos tres personas, dos observadores y un anotador, todos los observadores contaron con al menos tres años de experiencia. Los recorridos se llevaron a cabo entre las 07:00 y las 17:00hrs. Con base a la naturaleza del terreno los conteos se realizaron desde vehículos o a pie. Cada visita tuvo una duración de cinco días. Las especies se identificaron utilizando diferentes guías de campo (Howell \& Webb, 1995; NGS, 2002; van Perlo, 2006). Las observaciones fueron hechas con binoculares (8 y 10x) y telescopio (15-60x). En cada visita se censaron siempre las mismas áreas mediante recorridos preestablecidos al iniciar el trabajo, lo que permitió la comparación ente las abundancias vistas en los diferentes meses y proporcionó un estimado mínimo del número de aves que utilizaron el área.

La independencia de las abundancias de cada especie con respecto al mes y al sitio de observación se verificó mediante pruebas de $X^{2}$ múltiple ( $\left.a=0,05 ; Z a r, 1999\right)$.

\section{RESULTADOS}

Las abundancias de los Carraos no pudieron ser consideradas independientes del mes y del sitio de observación 
$\left(X_{28}^{2}=558,3 ; P<0,005\right)$. A lo largo de los ocho meses de muestreo se realizaron un total de 493 registros, que incluyeron 31 eventos de observación (tamaño promedio del grupo 15,9 aves). Se observaron desde aves solitarias hasta parvadas de 80 individuos. Espacialmente el sitio con mayor número de registros fue Las Cañadas (39\%), seguido de La Batanga (25\%), el sitio con menor número de observaciones fue Zoyata (6\%; Cuadro 1).

Temporalmente se observaron las menores abundancias en noviembre, números medios entre diciembre y febrero, los mayores números se registraron en marzo y abril, para disminuir drásticamente en mayo y junio (Cuadro 1). Sobresalen los registros de marzo en La Batanga, pues se observaron dos parvadas de 19 y 35 aves, similarmente en abril en Las Cañadas se registraron las tres bandadas más numerosas, de 40, 50 y 80 aves.

Por su parte, las abundancias del Gavilán Caracolero tampoco se consideraron independientes del mes y del sitio de observación $\left(X^{2}{ }_{14}=28,5, P<0,025\right)$. Para el Gavilán Caracolero en el mismo lapso de tiempo se realizaron 109 registros en tres zonas (Cuadro 2). Esta especie siempre fue observada de manera solitaria. El sitio con mayor

CUADRO 1

Registros de abundancia del Carrao (Aramus guarauna) realizados en Marismas Nacionales.

\begin{tabular}{|c|c|c|c|c|c|c|}
\hline \multicolumn{7}{|c|}{ Sitios } \\
\hline Mes & Zoquipan & La Batanga & Mexcaltitán & Las Cañadas & Zoyata & Total \\
\hline Noviembre & 2 & 1 & 3 & 0 & 0 & $6(1 \%)$ \\
\hline Diciembre & 3 & 15 & 0 & 10 & 5 & $33(7 \%)$ \\
\hline Enero & 10 & 10 & 7 & 5 & 7 & $39(8 \%)$ \\
\hline Febrero & 29 & 5 & 6 & 3 & 2 & $45(9 \%)$ \\
\hline Marzo & 9 & 61 & 41 & 0 & 6 & $117(24 \%)$ \\
\hline Abril & 2 & 22 & 10 & 174 & 2 & $210(42 \%)$ \\
\hline Mayo & 4 & 5 & 0 & 0 & 6 & $15(3 \%)$ \\
\hline Junio & 24 & 4 & 0 & 0 & 0 & $28(6 \%)$ \\
\hline Total & 83 (17\%) & $123(25 \%)$ & 67 (13\%) & 192 (39\%) & $28(6 \%)$ & 493 \\
\hline
\end{tabular}

Se indica el sitio y el mes de registro. Se incluye los totales por sitio y mes y sus porcentajes.

CUADRO 2

Registros de abundancia del Gavilán Caracolero (Rostrhamus sociabilis) realizados en Marismas Nacionales.

\begin{tabular}{lcccc}
\hline \multicolumn{5}{c}{ Sitios } \\
\hline \multicolumn{1}{c}{ Mes } & Zoquipan & La Batanga & Mexcaltitán & Total \\
Noviembre & 18 & 0 & 5 & $23(21 \%)$ \\
Diciembre & 7 & 0 & 0 & $7(6 \%)$ \\
Enero & 12 & 1 & 9 & $22(20 \%)$ \\
Febrero & 8 & 2 & 7 & $17(16 \%)$ \\
Marzo & 3 & 0 & 5 & $8(7 \%)$ \\
Abril & 10 & 0 & 6 & $16(15 \%)$ \\
Mayo & 12 & 0 & 1 & $13(12 \%)$ \\
Junio & 2 & 1 & 0 & $3(3 \%)$ \\
Total & $72(66 \%)$ & $4(4 \%)$ & $33(30 \%)$ & 109
\end{tabular}


número de registros fue Zoquipan, seguido de Mexcaltitán y por último La Batanga, con poco menos del 4\%. Temporalmente se observaron notorias oscilaciones, los números mayores (con poco más de 20 aves) se llevaron a cabo en noviembre y enero y los menores (menos de 10 aves) en diciembre, marzo y junio (Cuadro 2).

\section{DISCUSIÓN}

Pese a que no existe un seguimiento temporal de las especies de aves que utilizan los humedales nayaritas, al menos entre 1988 y 1999, el Carrao y el Gavilán Caracolero no se habían registrado en el estado (Escalante-Pliego, 1988; Cupul-Magaña, 1999), lo que aunado a las fechas de registro anteriores confirma la idea de su llegada reciente.

Como ha sido registrado en otros sitios (Angehr, 1999), el arribo de estas dos especies fue antecedido por la colonización de los cuerpos de agua por el caracol manzana, su principal fuente de alimento (Sykes et al., 1995; Bryan, 2002). Por observaciones personales se ha validado la presencia de este caracol a lo largo de los sitios de observación de las dos especies; es decir, Pomacea spp. ha continuado su expansión geográfica y actualmente incluye la mayor parte de los humedales salobres de Nayarit. Considerando a San Blas como el último punto de registro (Iliff \& Lovitch, 2007), actualmente los caracoles se localizan en humedales hasta $85 \mathrm{~km}$ más al norte (Zoyata, Fig. 1). Dadas las recientes observaciones de Carraos y Gavilanes Caracoleros, es posible que la llegada de los caracoles manzana también sea reciente, por lo que su arribo debió realizarse vía programas acuaculturales establecidos en la década de los años noventa del siglo pasado, como se ha indicado para Jalisco (Palomera-García et al., 2006).

El efecto de los caracoles manzana sobre los nuevos entornos que colonizan no ha sido documentado, sin embargo, dadas las abundancias observadas en diferentes puntos (e.g. Zoquipan), la presencia de esta especie debió modificar drásticamente el ensamble de especies bentónicas. Adicionalmente se sabe que algunas especies de caracol manzana pueden llegar a ser consideradas como plagas, con impactos negativos, tanto económicos (como en plantaciones de arroz), como ecológicos (competencia y herbivorismo; Halwart, 1994; Martín, Estebenet \& Cazzaniga, 2001). Lo anterior es producto de que este género presenta acelerados crecimientos individual y poblacional, que se incrementan en primavera y verano, con las llegadas de las primeras Iluvias y temperaturas calidas (Estebenet \& Cazzaniga, 1992). También se ha observado que su expansión, aunque puede ser lenta, se ve favorecida por la presencia de canales de irrigación (Watanabe et al., 2000) como los que se presentan en Marismas Nacionales.
También es interesante resaltar que los puntos de observación (ver Fig. 1) se apegan geográficamente a las observaciones previas mencionadas en la literatura (Iliff \& Lovitch, 2007), pues en los sitios sureños (Zoquipan, La Batanga y Mexcaltitán) se observaron las dos especies, mientras que en los sitios intermedios (Las Cañadas y Zoyata) sólo se observaron Carraos. En los norteños aún no se han registrado. Adicionalmente la presencia de las dos especies de interés está relacionada con la salinidad, pues en todos los casos se trató de cuerpos de agua con escasa salinidad y con presencia de lirio acuático y de tule, los preferidos por los caracoles manzana para su alimentación (Naranjo-García, 2003). De hecho los dos sitios intermedio (Fig. 1), donde no se observaron las especies de interés, corresponden a lagunas abiertas con salinidades elevadas y con escasa vegetación acuática.

Respecto al origen de las dos especies, dados los patrones temporales de aparición en el Pacífico mexicano (Howell, 1994, 2004; Hernández-Vázquez et al., 1999; Palomera-García et al., 2006; Iliff \& Lovitch, 2007), es muy probable que se trate de aves provenientes del sur de México (Chiapas y Oaxaca), sitios donde son especies comunes (Sykes et al., 1995; Bryan, 2002).

La llegada del Carrao y del Gavilán Caracolero a los humedales nayaritas no parece haber ocasionado competencia con otras especies de aves, probablemente debido a su alimentación especializada (caracol manzana). Sin embargo, innegablemente los cambios en el ensamble bentónico debieron afectar a algunas especies, se carece de la información previa requerida para profundizar a este respecto. En adición a corto plazo podría presentarse competencia por algún recurso, por ejemplo espacio o materiales para la construcción de nidos, si estas especies llagaran a anidar en la zona. Por otra parte actualmente no existen acciones por parte del Gobierno Mexicano que combatan la colonización del caracol manzana, al menos en parte por la falta de información sobre los invertebrados de zonas blandas de los humedales nayaritas, lo que imposibilita determinar incluso las zonas donde tales acciones debieran realizarse.

Ambas especies son consideradas como residentes, aunque al menos para la población de Carraos de Florida se ha indicado que pueden ser parcialmente migratorios (Bryan, 2002). Pese a lo anterior para ambas especies se han indicado movimientos asociados a los cambios en los niveles del agua (Sykes et al., 1995; Bennetts \& Kitchens, 1997; Bryan, 2002), es probable que las diferencias temporales en la utilización de las zonas de Marismas Nacionales obedezcan a este tipo de movimientos, ya que los dos meses de mayores registros (marzo y abril) coinciden con la época de estiaje y son los más alejados de la temporada 
de lluvias (INEGI, 2011), de tal forma pudiera haber una congregación de los individuos en las lagunas salobres de mayor tamaño, las visitadas, y una posterior dispersión relacionada con el inicio del período de lluvias en mayo (INEGI, 2011).

Los meses de observación incluyen la época reproductiva de ambas especies (Sykes et al., 1995; Bryan, 2002), lo que aunado al registro reciente de anidación de Gavilanes Caracoleros en Colima (Palomera-García et al., 2006), hacen pensar en su actual o próxima reproducción en Marismas Nacionales.

En suma Marismas Nacionales es un sistema de humedales ya ocupado regularmente por los Carraos y los Gavilanes Caracoleros, dada la previa llegada de los caracoles manzana y es muy probable su reproducción actual o en corto plazo en el área, lo que incrementa la importancia de la zona, al recordar que ambas especies se encuentran protegidas por el Gobierno Mexicano. Los cambios en la abundancia de los Carraos se pueden relacionar con los períodos de lluvias de las zonas dados los cambios en los niveles de agua. Además se considera que éstas especies continuarán expandiendo su intervalo de distribución a lo largo del Pacífico mexicano, al menos hasta la parte norte de Sinaloa, pues en esta zona disminuyen los ambientes con vegetación acuática y de agua dulce (García-Páez \& Cruz-Medina, 2009), lo que actuará como una barrera natural, que dificultará la colonización del caracol manzana.

\section{AGRADECIMIENTOS}

A Pronatura Noroeste oficina Tepic particularmente a Mauricio Cortés y César Rodríguez por su apoyo en el trabajo de campo. Este trabajo fue parcialmente apoyado por el Proyecto "Planificación de mecanismo para la conservación legal y restauración de las poblaciones de aves acuáticas migratorias invernantes en Marismas Nacionales, Nayarit, México", auspiciado por North American Wetland Conservation Act, agradecemos particularmente el apoyo de Ellen Murphy. También se recibió apoyo de Kennecott Utah Copper Corporation, and the Rio Tinto-BirdLife Program, por lo que se agradece a Jonathan Stacey e Ítala Yepes, al equipo del Secretariado de las Américas de BirdLife International y a los miembros de Linking Communities.

\section{REFERENCIAS}

Angehr, G. R. (1999). Rapid long-distance colonization of Lake Gatun, Panama, by Snail Kites. Wilson Bulletin, 111(2), 265-268.

Bennetts, R. \& Kitchens, W. (1997). Population dynamics and conservation of the Snail Kite in Florida: The importance of the spatial and temporal scale. Colonial Waterbirds, 20(2), 324-329.

Bryan, D. C. (2002). Limpkin (Aramus guarauna). En Poole, A. (Ed.), The Birds of North America, No. 627. Recuperado de http:// bna.birds.cornell.edu/bna/species/627. doi:10.2173/ bna.627.

Comisión Nacional del Agua (CONAGUA). (2004). Base de datos del Clima en Nayarit. Gerencia Estatal en Nayarit. México: Subgerencia Técnica. Departamento de Meteorología.

Comisión Nacional de Áreas Naturales Protegidas. (2005). Estudio previo justificativo para el establecimiento del Área $\mathrm{Na}$ tural Protegida. SEMARNAT. Recuperado de http://www. conanp.gob.mx./anp/consulta/

Cupul-Magaña, F. G. (1999). La laguna El Quelele, Nayarit, México, como hábitat de aves acuáticas. Ciencia y Mar, 3, 21-28.

Diario Oficial de la Federación. (DOF). (2010). Norma Oficial Mexicana NOM-059-ECOL-2010, Protección ambiental-especies nativas de México de flora y fauna silvestres categorías de riesgo y especificaciones para su inclusión, exclusión o cambio-lista de especies en riesgo. 30 dic. 2010. México, D.F. Recuperado de http://www.semarnat.gob.mx/temas/.../ NOM_059_SEMARNAT_2010.

Escalante-Pliego, P. (1988). Aves de Nayarit. México: Coordinación General de Enseñanza Superior.

Estebenet, A. L. \& Cazzaniga, N. J. (1992). Growth and demography of Pomacea canaliculata (Gastropoda: Ampullariidae) under laboratory conditions. Malacological Review, 25(1-2), 1-12.

García-Páez, F. \& Cruz-Medina, I. R. (2009). Variabilidad de la precipitación pluvial en la Región Pacífico Norte de México. Agrociencia, 43(1), 1-9.

García-Ulloa, M., Rammarine, W., Ponce-Palafox, J. T. \& GóngoraGómez, A. M. (2006). Efecto de la depuración en la biomasa del caracol Pomacea patula (Baker 1922) usando en índice de condición. AIA, 10(3), 69-80.

Gómez de Silva, H. (2002). México. North American Birds, 56(2), 228-232.

Halwart, M. (1994). The golden apple snail Pomacea canaliculata in Asian rice farming systems: Present impact and future threat. International Journal of Pest Management, 40(2), 199-206.

Hernández-Vázquez, S., Esparza, R., Durand, B. C. \& Alvarado, L. F. (1999). Primer registro del Carrao (Aramus guarauna) en la costa de Jalisco, México. Boletín Centro Investigaciones Biológicas Universidad de Zulia, 33(2), 93-99. 
Howell, S. N. G. (1994). Additional information on the birds of Colima and adjacent Jalisco, Mexico. Euphonia, 3(2), 33-54.

Howell, S. N. G. (2004). Further observations of birds from Colima and adjacent Jalisco, Mexico. Cotinga, 21, 38-43.

Howell, S. N. G. \& Webb, S. (1995). A Guide to the Birds of Mexico and Northern Central America. Oxford, USA: Oxford University Press

Iliff M. J. \& Lovitch, D. (2007). The Changing Seasons: Foods for thought. North American Birds, 61(2), 208-224.

Instituto Nacional de Estadística y Geografía (INEGI). (2000). Síntesis de información geográfica del Estado de Nayarit. México: Instituto Nacional de Estadística Geografía e Informática.

Instituto Nacional de Estadística y Geografía (INEGI). (2011). Anuario de estadísticas por entidad federativa 2011. México: Instituto Nacional de Estadística Geografía e Informática.

Kovacs, J. M., Zhang, C. \& Flores-Verdugo, F. J. (2008). Mapping the condition of mangroves of the Mexican Pacific using C-band ENVISAT ASAR and Landsat optical data. Ciencias Marinas, 34(4), 407-418.

Martín, P. R., Estebenet, A. L. \& Cazzaniga, N. J. (2001). Factors affecting the distribution of Pomacea canaliculata (Gastropoda: Ampullariidae) along its southernmost natural limit. Malacología 43(1/2), 13-23.

Molina, D. (2008). Aves de Humedales de la costa sur de Nayarit. (Tesis inédita de licenciatura). Instituto Tecnológico de Bahía de Banderas, La Cruz de Huanacaxtle, Nayarit, México.
Naranjo-García, E. (2003). Moluscos continentales de México: Dulceacuícolas. Revista Biología Tropical, 51 (3), 495-505.

National Geographic Society (NGS). (2002). Field guide to the birds of North America. National. Washington, D.C., USA: Geographic Society.

Ortega-Solís, L. E. (2011). Distribución especial y temporal de aves playeras invernantes en la Reserva de la Biosfera Marismas Nacionales, Nayarit. (Tesis inédita de licenciatura). Universidad Autónoma de Nayarit, Xalisco, Nayarit, México.

Palomera-García, C., Contreras-Martínez, S., Cruz-Rivera, B. Y., Villa Bonilla, B. \& Gómez-Llamas. J. C. (2006). Registros adicionales del Carrao (Aramus guarauna) en el Estado de Jalisco, México. Huitzil, 7(1), 23-26.

Sykes, Jr., P. W., Rodgers, Jr., J. A., \& Bennetts, R. E. (1995). Snail Kite (Rostrhamus sociabilis). En Poole, A. (ed), The Birds of North America. No. 171. Recuperado de http://bna.birds.cornell. edu/bna/species/171. doi: 10.2173/bna.171

Van Perlo, B. (2006). Birds of Mexico and Central America. Princeton, New Jersey, USA: Princeton University Press.

Watanabe, T., Tanaka, K., Higuchi, H., Miyamoto, K., Kiyonaga, T., Kiyota, H., Suzuki, Y. \& Wada, T. (2000). Emergence of the apple snail, Pomacea canaliculata, (Gastropoda: Ampullariidae), after irrigation in a paddy. Applied Entomology and Zoology, 35(1), 75-79.

Zar, J. H. (1999). Biostatistical Analysis. New Jersey, USA: PrenticeHall, Inc. 
